\section{Another two stings in WASP's tail}

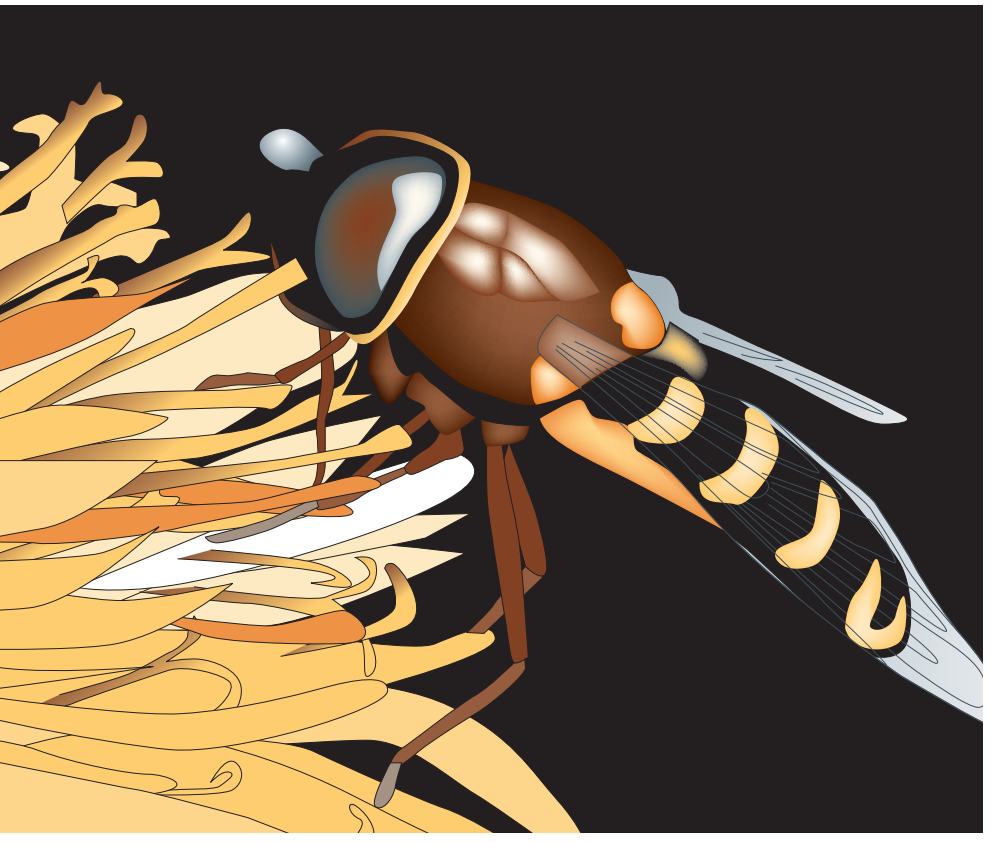

As direct activators of the Arp2/3 complex, Wiskott-Aldrich syndrome proteins (WASPs) link Cdc42 activation to the formation of actin filaments, and WASP phosphorylation on tyrosine (Y) 291 enhances WASP activity towards the Arp2/3 complex. In Molecular Cell, Torres and Rosen further explore Y291 phosphorylation, while Cory et al. identify two new WASP phosphorylation sites.

Autoinhibition of WASP - by the carboxy-terminal VCA (verprolin homology, central hydrophobic, and acidic) domain binding to the GTPase-binding domain (GBD) - is relieved by active $\mathrm{Cdc} 42$. Torres and Rosen investigated cooperation between Cdc42 and tyrosine kinases in WASP regulation. Y291 is in the GBD, within the fold of the autoinhibited domain, and so is only expected to be phosphorylated when autoinhibition is relieved. Indeed, adding the VCA domain inhibited Y291 phosphorylation; this was relieved by adding a mimic of GTPbound Cdc42. Similarly, the autoinhibited fold protected phosphoY291 (pY291)-WASP against tyrosine phosphatases. So, WASP Y291 phosphorylation seems to be altered only when activated Cdc42 is present, which, in the context of positive signalling, would allow WASP to remain phosphorylated long after the initial stimulus had subsided.

It has previously been proposed that Y291 phosphorylation might destabilize autoinhibition and constitutively activate WASP. Indeed, the pY291-GBD still bound VCA, but with a lower affinity - its basal activity towards the Arp2/3 complex, though, increased. Another consequence was that Src, through its Srchomology-2 (SH2) domain, could displace pY291-GBD from the VCA, which, again, increases Arp2/3 activity. Src kinase activity might also potentiate WASP activation by phosphorylating Y291.

Cory et al. identified two phosphorylation sites — serine (S) 483 and serine 484 - in the VCA domain and showed that these sites in WASP and its relative N-WASP are phosphorylated in various cell types. S483 is within a consensus casein kinase 2 (CK2) phosphorylation

\section{CALCIUM}

\section{A local store}

Free $\mathrm{Ca}^{2+}$ in the nucleus can regulate important functions such as gene transcription. But how is the nuclear level of free $\mathrm{Ca}^{2+}$ controlled? In the models that have been proposed so far, $\mathrm{Ca}^{2+}$ simply reaches the nuclear interior from the nuclear envelope (NE) by diffusion - a mechanism that would only allow the nucleus to be regulated uniformly by $\mathrm{Ca}^{2+}$. However, this is not what occurs, so could the nucleus have its own local $\mathrm{Ca}^{2+}$ store? Nathanson and colleagues now provide the answer to this question in Nature Cell Biology.

In SKHep1 epithelial cells, the authors used the endoplasmic reticulum (ER) dye ERTracker to detect a nucleoplasmic reticulum - a fine, branching intranuclear network that is continuous with the ER and the NE. They confirmed the presence of this structure by showing that the ER protein calreticulin was distributed in a reticular pattern in the nucleus, as well as in the cytosol, of SKHep1 cells.
Nathanson and co-workers next showed that fluorescent $\mathrm{Ca}^{2+}$ dyes also labelled this intranuclear network, and they used fluorescence recovery after photobleaching to confirm that the dyes were membraneenclosed, rather than membrane-bound. These results therefore show that there is a nuclear $\mathrm{Ca}^{2+}$-storing network that is continuous with the ER and the NE.

Inositol-1,4,5-trisphosphate (InsP $\mathrm{P}_{3}$ ) receptors mediate $\mathrm{Ca}^{2+}$ signalling in SKHep1 cells, and the authors found that the type II Ins $\mathrm{P}_{3}$ receptor isoform is enriched in the nucleus of these cells. This isoform is expressed, in part, along the nucleoplasmic reticulum, but what do these intranuclear Ins $\mathrm{P}_{3}$ receptors do?

The authors developed a new technique to answer this question - a technique that allowed them to photorelease intranuclear nitrophenylethyl ester (NPE)-caged Ins $\mathrm{P}_{3}$ in a highly localized fashion in individual cells using two-photon excitation. When they photoreleased InsP ${ }_{3}$ within $1 \mu \mathrm{M}$ of the nucleoplasmic reticulum, they detected small increases in $\mathrm{Ca}^{2+}$ that began at the nucleoplasmic reticulum and were greatest at the site of InsP ${ }_{3}$ release. These data allowed them to conclude that "...the nucleoplasmic reticulum is an $\mathrm{InsP}_{3}$-gated calcium store that can give rise to local calcium signals in the nuclear interior".

Finally, Nathanson and colleagues monitored how the distribution of protein kinase $\mathrm{C}-\gamma($ PKC- $\gamma)$, which contains a $\mathrm{Ca}^{2+}$-sensitive regulatory domain, is affected by $\mathrm{Ca}^{2+}$. They photoreleased $\mathrm{Ca}^{2+}$ in either the nucleus or the cytosol and examined the effect on the distribution of green fluorescent protein (GFP)-PKC- $\gamma$. They found that nuclear $\mathrm{Ca}^{2+}$ signals altered the distribution of nuclear, not cytosolic, GFP-PKC- $\gamma$ and vice versa, which indicates that nuclear and cytosolic $\mathrm{Ca}^{2+}$ signals can have effects that are independent of one another.

This work has therefore shown that "...the nucleus contains a nucleoplasmic reticulum with the capacity to regulate calcium signals in localized subnuclear regions". This discovery potentially explains how the nucleus can regulate several independent $\mathrm{Ca}^{2+}$-dependent processes simultaneously, and might have revealed a new layer of $\mathrm{Ca}^{2+}$ control.

\section{(2) References and links} Rachel Smallridge

ORIGINAL RESEARCH PAPER Echevarria, W. et al.

Regulation of calcium signals in the nucleus by a nucleoplasmic reticulum. Nature Cell Biol. 5, 440-446 (2003) WEB SITE

Michael Nathanson's laboratory:

http://info.med.yale.edu/intmed/digdis/otherpages/facsum $\mathrm{m} \cdot \mathrm{html} /$ nathanson.html 
site, and CK2 inhibitors decrease endogenous levels of pS483/pS484WASP. Cory et al. also showed that S483 and S484 are substrates for CK2 in vitro. S483/S484 phosphorylation enhanced VCA-Arp2/3 binding, and phosphorylation of S483/ S484 enhanced actin polymerization that was induced by activated Cdc42 or by a Y291 mutation that renders WASP constitutively active. S483/S484 phosphorylation, therefore, seems to be important for optimal functioning of activated WASP.

So, as Cory et al. conclude, “...post-translational modification of WASP is important in its regulation $\ldots$ and it will be of great interest to determine the interplay between phosphorylation and other cellular regulators of WASP function".

Katrin Bussell

\section{(2) References and links} ORIGINAL RESEARCH PAPERS Torres, E. \& Rosen, M. K. Contingent phosphorylation/ dephosphorylation provides a mechanism of molecular memory in WASP. Mol. Cell 11, 1215-1227 (2003) | Cory, G. O. C. et al. Phosphorylation of the WASP-VCA domain increases its affinity for the Arp2/3 complex and enhances actin polymerization by WASP. Mol. Cell 11, 1229-1239 (2003)

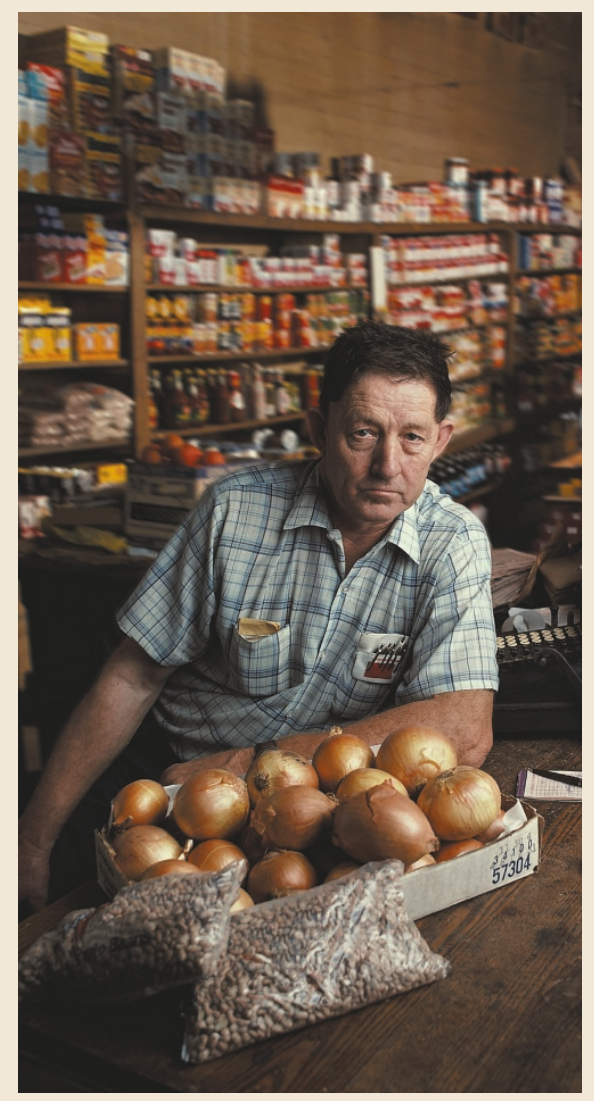

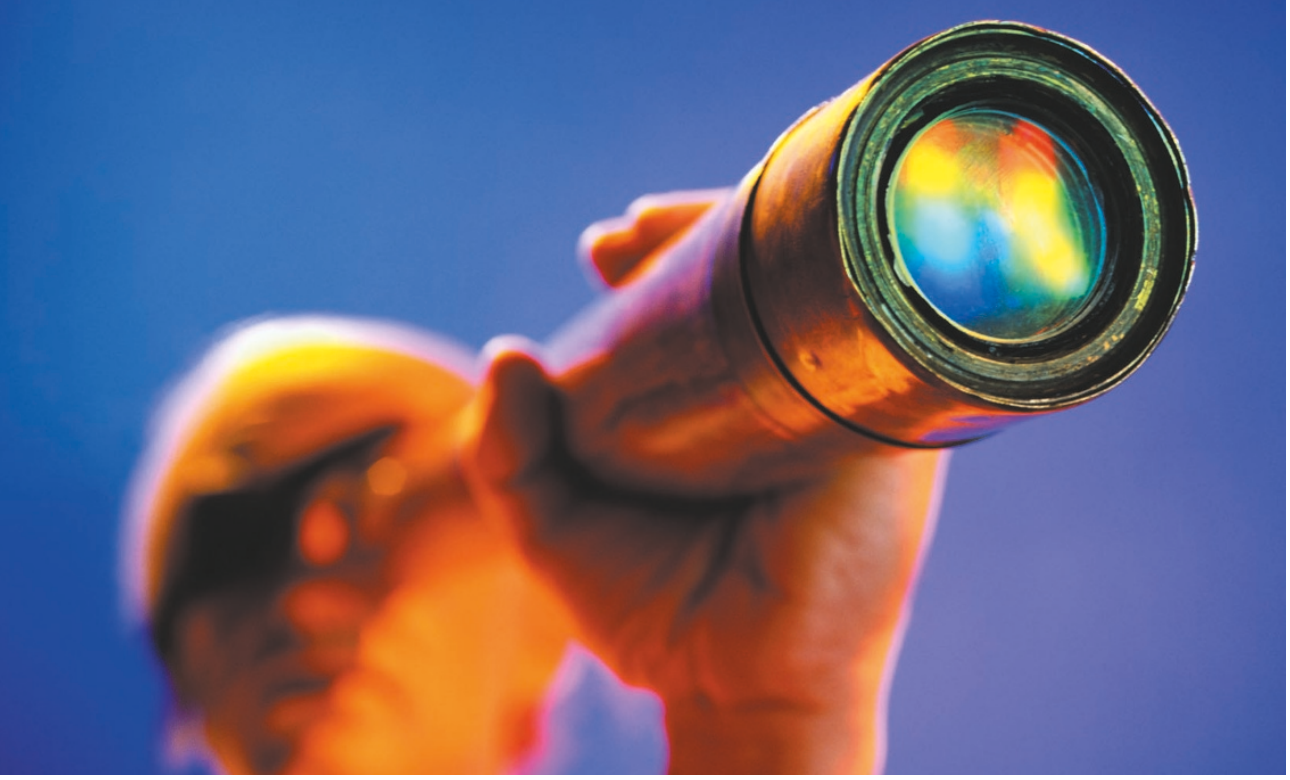

\section{PROTEOGLYCANS}

\section{A close-up of chondroitin}

Chondroitin and heparan sulphate, two types of glycosaminoglycan, are well-known sugar polymers in animals. Much more is known about heparan sulphate's involvement during development compared with chondroitin sulphate's, but, thanks to two reports in Nature, chondroitin is at last in the spotlight, and is now shown to be involved in Caenorhabditis elegans cytokinesis and morphogenesis.

The so-called squashed vulva ( $s q v)$ genes are important for embryonic development and postembryonic vulval morphogenesis, and seven of these are known to regulate the biosynthesis of chondroitin and heparan sulphate. Hwang et al. cloned the eighth sqv gene, $s q v-5$, corresponding to the gene sequence T24D1.1, which Mizuguchi et al. also cloned in a separate study.

The protein encoded by T24D1.1 is similar to both human chondroitin synthase and chondroitin $\mathrm{N}$-acetylgalactosaminyltransferase, which are needed for the initiation and elongation of chondroitin chains, respectively. In C. elegans, though, SQV-5 - or chondroitin synthase (ChSy), as Mizuguchi et al. refer to it - carries out both functions; protein extracts prepared from worms homozygous for a $s q v-5$ null allele lacked both activities. And both groups reported a marked reduction in the levels of chondroitin in the absence of $s q v-5 / C h S y$.

Both groups used conventional mutations or RNA interference (RNAi) - by feeding the worms dsRNA - to suppress sqv-5/ChSy expression. RNAi caused weaker defects compared with conventional mutations; $\sim 90 \%$ of embryos from RNAi-treated worms died whereas all embryos of conventional mutants died. Mizuguchi et al. also noticed that $60 \%$ of the survivors of RNAi treatment showed poor gonad formation and laid few, morphologically abnormal eggs. Hwang et al. reported that RNAi treatment reduced vulval extracellular spaces.

A closer look by Mizuguchi et al., using fourdimensional microscopy, showed severe cell-division defects. Embryonic cell division seemed to progress and then reverse (from 2 to 4 , to 6 cells, to 4 , to 6 cells, and so on), apparently as a result of incomplete cytokinesis. Eggs laid after longer RNAi treatment, which had even less chondroitin, underwent normal nuclear division, but failed to undergo cytokinesis altogether. So, if chondroitin is required for normal embryonic cell division and cytokinesis, it follows, then, that treating normal embryonic cells with chondroitinase should also induce similar phenotypes — which was indeed the case.

Consistent with a role for chondroitin in cytokinesis, early embryogenesis and morphogenesis, Mizuguchi et al. showed that chondroitin was present in the oocytes, the uterus, spermatheca and fertilized egg shells. The cell surfaces of early embryos expressed high levels, too. Using anti-SQV-5 antibodies, Hwang et al. observed punctate staining in the cytoplasm of the vulva, uterus and oocytes, similar to the staining pattern that had previously been observed for SQV-1 and SQV-7. So Hwang et al. propose that the chondroitin biosynthetic steps that are catalysed by these SQV proteins all occur in the same subcellular compartment - most probably the Golgi apparatus.

Hwang et al. also propose that chondroitin's ability to interact with water, which would generate osmotic pressure, could well be responsible for its ability to expand extracellular space, on the basis that observed defects in the first embryonic cytokinesis and vulval morphogenesis occur concomitantly with a reduction in extracellular matrix size. Mizuguchi et al. also suggest that the role of chondroitin in cell division and cytokinesis could be a structural one, but raise the possibility that an unidentified chondroitin-dependent signalling event might be necessary for the completion of cytokinesis, and indicate that the possible relationship of chondroitin with components of the cell cycle and cytoskeleton requires investigation.

Katrin Bussell

\section{(2) References and links}

ORIGINAL RESEARCH PAPERS Hwang, H.-Y. et al. Caenorhabditis elegans early embryogenesis and vulval morphogenesis require chondroitin biosynthesis. Nature 423, 439-443 (2003) |

Mizuguchi, S. et al. Chondroitin proteoglycans are involved in cell division of Caenorhabditis elegans. Nature 423, 443-448 (2003) 